When the liquid compound was treated with an alcoholic solution of potash, it was also resolved into benzoate of potash, chloride of potassium, and a similar resinons matter, the quantity of which appeared to be even greater than that yielded by the solid compound.

Neutral benzoate of lead, formed by adding a solution of benzoate of soda to acetate of lead, was also subjected to destructive distillation. A small quantity of benzoic acid came over mixed with a little of an aromatic oil, the smell of which was quite different from that yielded by benzoate of copper. I was unable, however, to detect the presence of any neutral crystalline body.

Glasgow, May 12, 1845.

III. On the Aberration of Light. By G. G. Stoкes, M.A., Fellow of Pembroke College, Cambridge*.

THE general explanation of the phænomenon of aberration is so simple, and the coincidence of the value of the velocity of light thence deduced with that derived from observations of eclipses of Jupiter's satellites so remarkable, as to leave no doubt on the mind as to the truth of that explanation. But when we examine the cause of the phænomenon more closely, it is far from being so simple as it appears at first sight. On the theory of emissions, indeed, there is little difficulty; and it would seem that the more particular explanation of the cause of aberration usually given, which depends on the consideration of the motion of a telescope as light passes from its object-glass to its cross wires, has reference especially to this theory; for it does not apply to the theory of undulations, unless we make the rather startling hypothesis, that the luminiferous ather passes freely through the sides of the telescope and through the earth itself. The undulatory theory of light, however, explains so simply and so beautifully the most complicated phænomena, that we are naturally led to rega. I aberration as a phænomenon unexplained by it, but not incompatible with it.

The object of the present communication is to attempt an explanation of the cause of aberration which shall be in accordance with the theory of undulations. I shall suppose that the earth and planets carry a portion of the gether along with them so that the æther close to their surfaces is at rest relatively io those surfaces, while its velocity alters as we recede

* Comnunicated by the Author. 
from the surface, till, at no great distance, it is at rest in space. According to the undulatory theory, the direction in which a heavenly body is seen is normal to the fronts of the waves which have emanated from it, and which have reached the neighbourhood of the observer, the æther near him being supposed to be at rest relatively to him. If the æther in space were at rest, the front of a wave of light at any instant being given, its front at any future time could be found by the method explained in Airy's Tracts. If the æther were in motion, and the velocity of propagation of light were infinitely small, the wave's front would be displaced as a surface of particles of the æther. Neither of these suppositions is however true, for the æther moves while light is propagated through it. In the following investigation I suppose that the displacements of a wave's front in an elementary portion of time due to the two causes just considered take place independently.

Let $u, v, w$ be the resolved parts along the rectangular axes of $x, y, z$, of the velocity of the particle of ather whose coordinates are $x, y, z$, and let $\mathrm{V}$ be the velocity of light supposing the æether at rest. In consequence of the distance of the heavenly bodies, it will be quite unnecessary to consider any waves but those which are plane, except in so far as they are distorted by the motion of the æther. Let the axis of $z$ be taken in, or nearly in the direction of propagation of the wave considered, so that the equation to the wave's front at any time will be

$$
z=\mathrm{C}+\mathrm{V} t+\zeta, \cdot . \cdot . \cdot .
$$

$\mathrm{C}$ being a constant, $t$ the time, and $\zeta$ a small quantity, a function of $x, y$ and $t$. Since $u, v, w$ and $\zeta$ are of the order of the aberration, their squares and products may be neglected.

Denoting by $\alpha, \beta, \gamma$ the angles which the normal to the wave's firont at the point $(x, y, z)$ makes with the axes, we have, to the first order of approximation,

$$
\cos \alpha=-\frac{d \zeta}{d x}, \quad \cos \beta=-\frac{d \zeta}{d y}, \quad \cos \gamma=1 ; . .
$$

and if we take a length $V d t$ along this normal, the co-ordinates of its extremity will be

$$
x-\frac{d \zeta}{d x} \mathrm{~V} d t, \quad y-\frac{d \zeta}{d y} \mathrm{~V} d t, z+\mathrm{V} d t
$$

If the ather were at rest, the locus of these extremities would be the wave's front at the time $t+d t$, but since it is in motion, the co-ordinates of those extremities must be further in-. creased by $u d t$, $v d t$, wdt. Denoting then by $x^{\prime}, y^{\prime}, z^{\prime}$ the co-ordinates of the point of the wave's front at the time $t+d t$, 
Mr. G. G. Stokes on the Aberration of Light.

which corresponds to the point $(x, y, z)$ in its front at the time $t$, we have

$$
\begin{gathered}
x^{\prime}=x+\left(u-\mathrm{V} \frac{d \zeta}{d x}\right) d t, \quad y^{\prime}=y+\left(v-\mathrm{V} \frac{d \zeta}{d y}\right) d t, \\
z^{\prime}=z+(w+\mathrm{V}) d t ;
\end{gathered}
$$

and eliminating $x, y$ and $z$ from these equations and (1.), and denoting $\zeta$ by $f(x, y, t)$, we have for the equation to the wave's front at the time $t+d t$,

$$
\begin{aligned}
& z^{\prime}-(w+\mathrm{V}) d t=\mathrm{C}+\mathrm{V} t \\
& +f\left\{x^{t}-\left(u-\mathrm{V} \frac{d \zeta}{d x}\right) d t, \quad y^{t}-\left(v-\mathrm{V} \frac{d \zeta}{d y}\right) d t, t\right\},
\end{aligned}
$$

or, expanding, neglecting $d t^{2}$ and the square of the aberration, and suppressing the accents of $x, y$ and $z$,

$$
z=\mathrm{C}+\mathrm{V} t+\zeta+(w+\mathrm{V}) d t . . .
$$

But from the definition of $\zeta$ it follows that the equation to the wave's front at the time $t+d t$ will be got from (1.) by putting $t+d t$ for $t$, and we have therefore for this equation,

$$
z=\mathrm{C}+\mathrm{V} t+\zeta+\left(\mathrm{V}+\frac{d \zeta}{d t}\right) d t . \quad . .
$$

Comparing the identical equations (3.) and (4.), we have

$$
\frac{d \zeta}{d t}=w \text {. }
$$

This equation gives $\zeta=\int w d t$ : but in the small term $\zeta$ we may replace $\int w d t$ by $\frac{1}{\mathrm{~V}} \int w d z$; this comes to taking the approximate value of $z$ given by the equation $z=\mathrm{C}+\mathrm{V} t$, instead of $t$, for the parameter of the system of surfaces formed by the wave's front in its successive positions. Hence equation (1.) becomes

$$
z=\mathrm{C}+\mathrm{V} t+\frac{1}{\mathrm{~V}} \int w d z .
$$

Combining the value of $\zeta$ just found with equations (2.), we get, to a first approximation,

$$
\alpha-\frac{\pi}{2}=\frac{1}{\mathrm{~V}} \int \frac{d w}{d x} d z, \quad \beta-\frac{\pi}{2}=\frac{1}{\mathrm{~V}} \int \frac{d w}{d y} d z,
$$

equations which might very easily be proved directly in a more geometrical manner.

If random values are assigned to $u, v$ and $w$, the law of aberration resulting from these equations will be a complicated 
one; but if $u, v$ and $w$ are such that $u d x+v d y+w d z$ is an exact differential, we have

$$
\frac{d w}{d x}=\frac{d u}{d z}, \quad \frac{d w}{d y}=\frac{d v}{d z}
$$

whence, denoting by the suffixes 1,2 the values of the variables belonging to the first and second limits respectively, we obtain

$$
\alpha_{2}-\alpha_{1}=\frac{u_{2}-u_{1}}{\mathrm{~V}}, \quad \beta_{2}-\beta_{1}=\frac{v_{2}-v_{1}}{\mathrm{~V}} . .
$$

If the motion of the æther be such that $u d x+v d y+w d z$ is an exact differential for one system of rectangular axes, it is easy to prove, by the transformation of co-ordinates, that it is an exact differential for any other system. Hence the formulæ (6.) will hold good, not merely for light propagated in the direction first considered, but for light propagated in any direction, the direction of propagation being taken in each case for the axis of $z$. If we assume that $u d x+v d y+w d z$ is an exact differential for that part of the motion of the rether which is due to the motions of translation of the earth and planets, it does not therefore follow that the same is true for that part which depends on their motions of rotation. Moreover, the diurnal aberration is too small to be detected by observation, or at least to be measured with any accuracy, and I shall therefore neglect it.

It is not difficult to show that the formulæ (6.) lead to the known law of aberration. In applying them to the case of a star, if we begin the integrations in equations (5.) at a point situated at such a distance from the earth that the motion of the ather, and consequently the resulting change in the direction of the light, is insensible, we shall have $u_{1}=0, v_{1}=0$; and if, moreover, we take the plane $x z$ to pass through the direction of the earth's motion, we shall have

and

$$
v_{2}=0, \quad \beta_{2}-\beta_{1}=0 \text {, }
$$

$$
\alpha_{2}-\alpha_{1}=\frac{u_{2}}{\mathrm{~V}}
$$

that is, the star will appear to be displaced towards the direction in which the earth is moving, through an angle equal to the ratio of the velocity of the earth to that of light, multiplied by the sine of the angle between the direction of the earth's motion and the line joining the earth and the star.

In considering the effect of aberration on a planet, it will be convenient to divide the integrations in equation (5.) into three parts, first integrating from the point considered on the surface of the planet to a distance at which the motion of the 
æther may be neglected, then to a point near the earth where we may still neglect the motion of the æther, and lastly to the point of the earth's surface at which the planet is viewed. For the first part we shall have $u_{2}=0, v_{2}=0$, and $u_{1}, v_{1}$ will be the resolved parts of the planet's velocity. The increments of $\alpha$ and $\beta$ for the first interval will be, therefore, $-\frac{u_{1}}{V},-\frac{v_{1}}{V}$. For the second interval $\alpha$ and $\beta$ will remain constant, while for the third their increments will be $\frac{u_{2}}{\mathrm{~V}}, \frac{v_{2}}{\mathrm{~V}}$, just as in the case of a star, $u_{2}$ and $v_{2}$ being now the resolved parts of the earth's velocity.

Fig. 1.

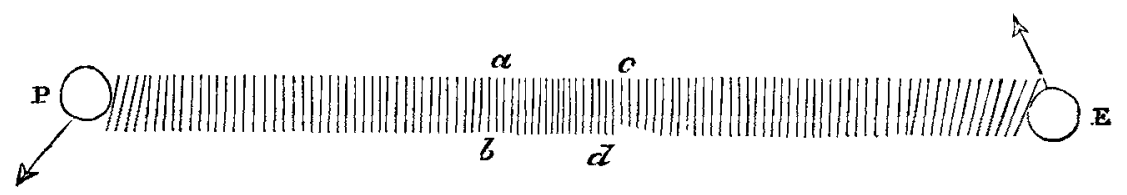

Fig. 2.

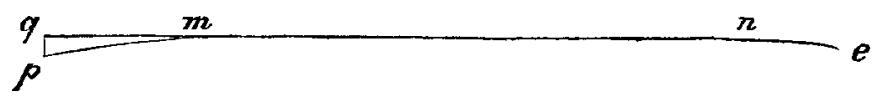

Fig. 1 represents what is conceived to take place. $P$ is the planet in the position it had when the light quitted it; E the earth in the position it has when the light reaches it. The lines $a b, c d$, \&c. represent a small portion of a wave of light in its successive positions. The arrows represent the directions in which $\mathrm{P}$ and $\mathrm{E}$ may be conceived to move. The breadth $a b$ is supposed to be comparable to the breadth of a telescope. In fig. $2, p m n e$ represents an orthogonal trajectory to the surfaces $a b, c d, \& c . ; p$ is the point of the planet from which the light starts, $e$ the point of the earth which it reaches. The trajectory $p$ in $n e$ may be considered a straight line, except near the ends $p$ and $e$, where it will be a little curved, as from $p$ to $m$ and from $e$ to $n$. The curvature at $e$ will have the same effect on the apparent position of the planet as it would have on that of a star in the same direction: as to the curvature at $p$, if we draw $p q$ perpendicular to $m n$ produced, the curvature will have the effect of causing $p$ to be seen as if it were at $q$. Now the angle between the tangents at $p$ and $m$ being that through which a star in the direction of $e$ is displaced by aberration to an observer at $p$, and the di-

- The lines towards $\mathbf{P}$ in fig. 1. should lean in the opposite direction. 
stance $p m$ being by hypothesis small (two or three radii of the planet suppose), it follows that the angle peq is extremely small, and may be neglected. Hence a planet will appear to be displaced from the position which it had when the light left it, just as a star in the same direction is displaced. But besides this, the planet has moved from $\mathrm{P}$ while the light has been travelling to $\mathrm{E}$. These two considerations combined lead to the formula for aberration, which is applicable to the planets, as is shown in treatises on astronomy. The same reasoning which applies to a planet will apply equally to the sun, the moon, or a comet.

To give an idea of the sort of magnitudes neglected in neglecting $p q$, suppose $p m$ equal to the diameter of $\mathrm{P}$, and suppose the curvature from $p$ to $m$ uniform. Let $r$ be the radius of $P, v$ its velocity, and $R$ the distance $P E$. The greatest possible value of the angle between the tangents at $p$ and $m$ is $\stackrel{v}{\tilde{V}^{*}}$ In this case we should have $\angle p e q=\frac{v r}{\mathrm{VR}}=\frac{v}{\mathrm{~V}} \mathrm{D}, \mathrm{D}$ being the semidiameter of $P$ as seen from $E$. Hence the angle peq must be very much greater for the moon than for any other body of the solar system; for in the case of the planets the value of $v$ is in no instance double its value for the earth or moon, while their discs are very small compared with that of the moon; and in the case of the sun, although its disc is about as large as that of the moon, its velocity round the centre of gravity of the solar system is very smail. It would indeed be more correct to suppose the sun's centre absolutely at rest, since all our measurements are referred to it, and not to the centre of gravity of the solar system. Taking then the case of the moon, and supposing $\frac{v}{\mathrm{~V}}=\frac{20^{\prime \prime}}{180^{\circ}} \pi, \mathrm{D}=15^{\prime}$, we find that the angle $p e q$ is about $\frac{1}{11}$ th of a second, an insensible quantity.

If we suppose the whole solar system to be moving in space with a velocity comparable with that of the earth round the sun, it follows from the linearity of the equations employed, that we may consider this motion separately. It is easy to show, that as far as regards this motion, the sun, moon, and planets will come into the positions in which they are seen just at the instant that the light from them reaches the earth. With respect to the stars also, that part of the aberration which varies with the time of year, the only part which can be observed, will not be affected. If we suppose the æether which fills the portion of space occupied by the solar system to be moving in a current, with a velocity comparable with that of the earth in its orbit, the result will still be the same. For if 
we suppose a velocity equal and opposite to that of the æther to be impressed, both on the æther and on the bodies of the solar system, the case is reduced to that of the solar system moving through the rether supposed to be at rest.

\section{On the Structure of Electro-precipitated Metals.} by Warren De la Rue, Esq.*

THE following observations, being the result of an extensive series of experiments on the practical application of the processes of electro-metallurgy, will, I am induced to believe, be acceptable to the Chemical Society.

The various appearances of the metallic deposit are familiar to all manipulators in electro-metallurgy, and are distinguished by the names crystalline, lesser crystalline, malleable, sandy and spongy; the latter being produced by an excess of power in the battery, the first by too small a power in relation to the strength of the solution operated on. All these deposits are however merely modifications of each other, they are essentially crystalline, and even the malleable, or in other words the most cohesive, is very inferior in strength to metals wrought by the processes in ordinnry use.

The malleable is that deposit usually required; yet, even with all the art of a practised electro-metallurgist, it is difficult for a lengthened period to obtain it, inasmuch as the power of the lattery, the temperature of the air, and consequently the conducting power of the fluids composing the circuit are constantly changing their relation to the strength of the electrolyte to be decomposed. There are other causes presently to be considered which also play an important part in producing these difficulties, and which we shall better understand by considering what effect the form of the matrix and the nature of its original surface have on the resulting precipitnte.

It is well known to persons conversant with the precipitation of metals from their respective solutions by means of voltaic electricity, that these solutions become exhausted of the metal at the cathode to such an extent, that if we place the cathode on the surface of the liquid all action after a short time ceases : the exhausted liquid being specifically lighter, no mechanical transfer of fresh liquid takes placet, and conse-

* Communicated by the Chemical Society; having been read February $17,1845$.

+ Professor Daniell and Dr. Miller, in a paper on the electrolysis of secondary compounds, have entered into the investigation of these phænomenn. 